\title{
WALKING TIME MEASURES FOR EVALUATING OA OF THE KNEE
}

\section{RELIABILITY AND SENSITIVITY \\ OF SELF-PACED WALKING TIME FOR ASSESSING FUNCTIONAL CAPACITY IN PERSONS WITH OSTEOARTHRITIS OF THE KNEE}

\author{
by $R$ Marks, Osteoarthritis Research \\ Centre, Toronto
}

\section{INTRODUCTION}

Although kinematic measures of walking time continue to be used for judging the efficacy of treatments for osteoarthritis $(\mathrm{OA})$ of the Knee ${ }^{1-7}$, no study to date has specifically investigated whether these measurements are reliable and sensitive to change in this patient population. Given the constantly fluctuating symptomatology of $\mathrm{OA}^{8}$, it is of utmost importance to ensure that interval changes in walking time can be confidently attributed to a true change in clinical status of the patient $t^{9}$. It is equally important to ensure that walking. time measurements designed to evaluate the efficacy of treatments for this condition are sensitive indicators of a change in functional status.

The purpose of this study was to examine the reliability of measurements of walking time for persons with stable knee OA over a six week period. A second purpose was to aid the clinician and/or investigator in determining whether measurements of walking time would be sufficiently sensitive for determining the efficacy of treatments other than surgery for patients with knee OA.

\section{PATIENTS AND METHODS}

\section{Patients}

The patients in this prospective study were 10 women aged $54-76$ years (mean 65.90 approx 8.34 ), height $155-167 \mathrm{~cm}$ (mean 161.9 approx 3.31) and weight 63$113 \mathrm{~kg}$ (mean 76.01 approx 15.28) who fulfilled the clinical and radiological criteria of the American College of Rheumatology (ARA) for the diagnosis of OA of the knee $^{11}$. In addition, three patients, age range 53-62 years, who fulfilled the ARA criteria were studied before and after completion of a six week isometric quadriceps strength training program. At study entry all participants complained of pain on walking. Fifty per cent were receiving nonsteroidal anti-inflammatory drugs for their arthritis and none used walking aids. As indicated by an average score of 12.80 approx 3.11 on The Lequesne Index of Disease Severity of Knee OA or ISK ${ }^{12}$, the cohorts studied were severely handicapped. The protocol was approved by a University Ethics Committee and all participants provided informed consent.

\section{Procedures}

The walking time measurements were carried out indoors on level ground using a stop watch. To allow a subject to walk at constant speed on the walkway and to control for the effects of acceleration and deceleration, subjects were instructed to walk with ordinary shoes at their normal comfortable walking pace for a distance of $3 \mathrm{~m}$ before walking time was recorded. The standardised procedures were carried out by the same observer six weeks later under the same theoretical no change conditions for the control group and after the six weeks of exercises for the experimental group.

\section{Statistical methods}

The statistics used to describe the data were the mean and standard deviation (SD). Reliability (R) was computed with intraclass correlation coefficients (ICCs) $(1,1)^{14}$. In addition, the standard error of the mean or SEM which represents the 68 percent confidence interval about the mean was computed from the reliability data as suggested by Hayes ${ }^{15}$. To assess the statistical significance of learning on the repeated measurements, a dependent ttest was used.

Differences in walking time, maximal isometric quadriceps torque and ISK scores at baseline and after six weeks of quadriceps strength training were expressed as a percentage of the baseline scores (see Table I). Pre- and post-exercise data were also compared using paired $t$ tests and Pearson correlations.

The level of significance for the analyses was set at $P$.

\section{ABSTRACT}

This study assessed the test-retest reliability and the sensitivity of selfpaced walking time measurements for evaluating the functional performance of persons with knee osteoarthritis (OA). The $13 \mathrm{~m}$ timed walking tests were carried out on two separate occasions over a six week period for 10 patients using a standardised protocol. The same measurements were recorded also for three additional patients before and after a six week quadriceps strength training regimen. As indicated by an intraclass correlation coefficient of 0.83 with no significant intersession test differences for the group $(\mathrm{P}=0.98)$, the gait measurements were reliable. However, despite marked increase in knee extensor strength and significant subjective improvements in walking time for the three experimental subjects were smaller than the error of measurement. The findings suggest that regardless of reliability, measurements of self-paced level walking time might not be sufficiently sensitive for evaluating longitudinal changes in functionalperformance in this patient population.

\section{RESULTS}

\section{Reliability}

The means, standard deviations and the 95 percent confidence intervals about the mean calculated for the walking tests are reported in Table II (page 7). As shown, the ICC or R statistic for the tests was 0.83 with a SEM of 1.50 seconds. As indicated by a Student's t-test there was no systematic learning effect with repeated testing.

\section{Sensitivity}

Although accompanied by a 4-8 percent improvement in walking time after exercise which correlated positively with individual increases of quadriceps strength $(r=0.90)$, this improvement $w$ as not significant $(t=2.05, p=0.17)$ and remained within the possible error of measurement of 1.50 seconds (see Table I page 7). The ISK scores which were also positively correlated with the walking time scores post-intervention $(r=0.86)$ were, however, significantly improved $(\mathrm{t}-8.66, \mathrm{p}=0.01)$. 


\section{MEDIO - TRONICS (Pty) LTD}

SPECIALIST SUPPLIERS TO THE PHYSIOTHERAPIST
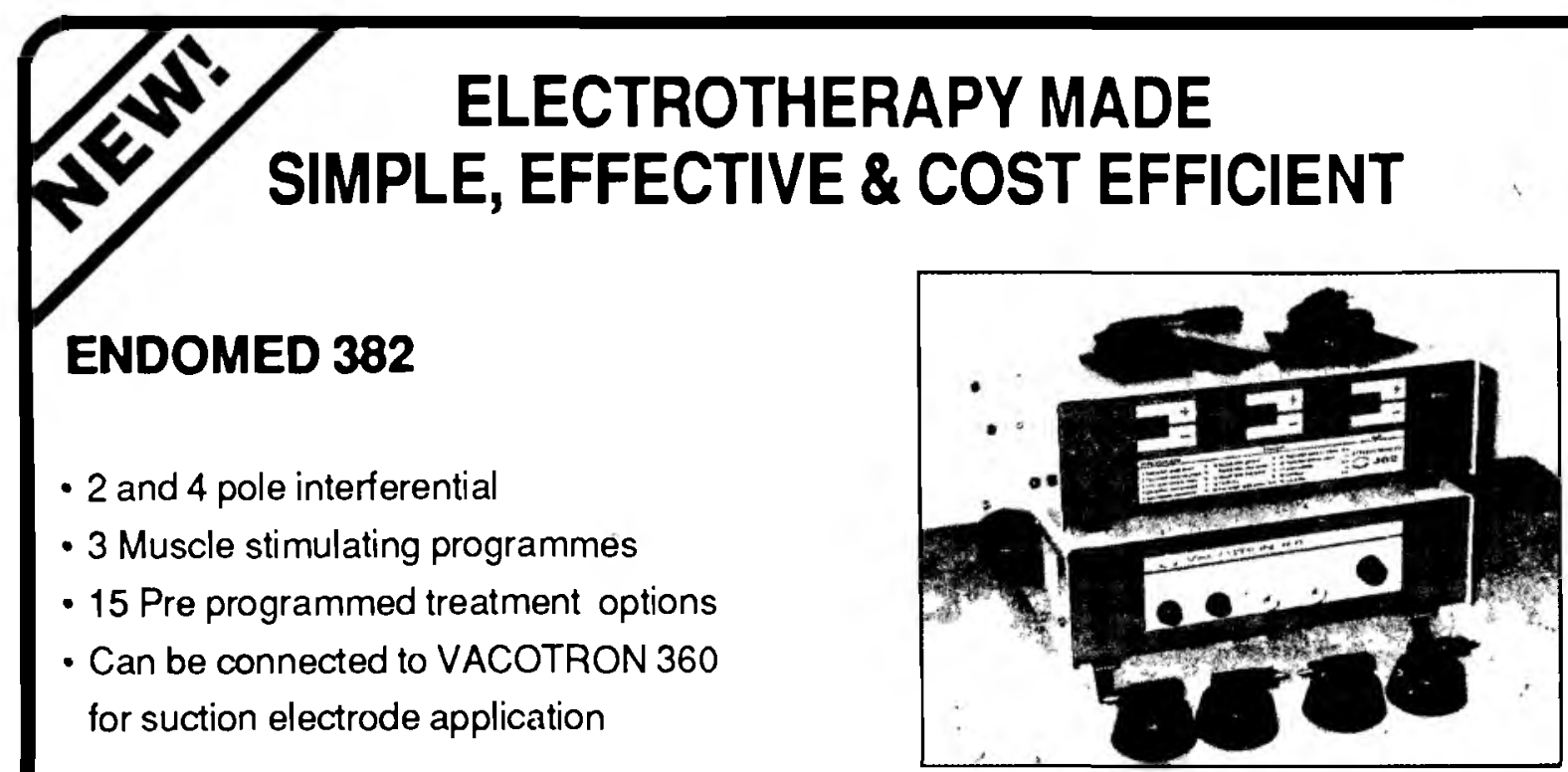

\section{SONOPULS $390-1 / 3$}

- $1 \mathrm{MHz}$ or $3 \mathrm{MHz}$ ultrasound units

- Continuous or pulsed ultrasound

- 10 additional pre-programmed treatment options

- Ultrasound heads have contact control

- Unit complete with small or large ultrasound head

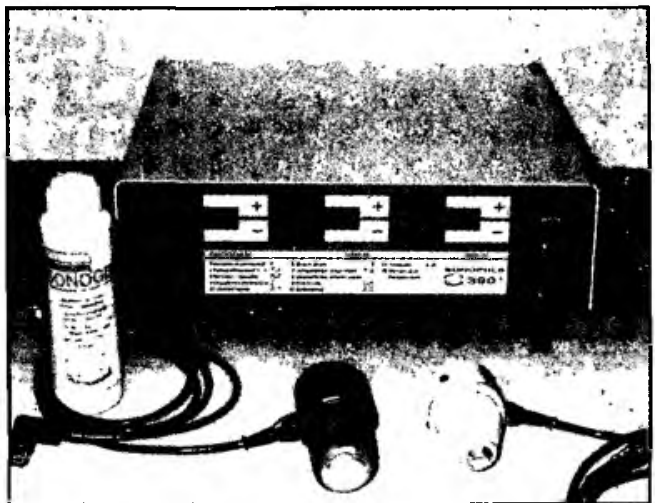

\section{ENDOMED 381 AC}

- 2 Pole interferential therapy

- 5 TENS currents

- 3 muscle stimulating programmes

- 15 pre-programmed treatment options

- can be connected to VACOTRON 360
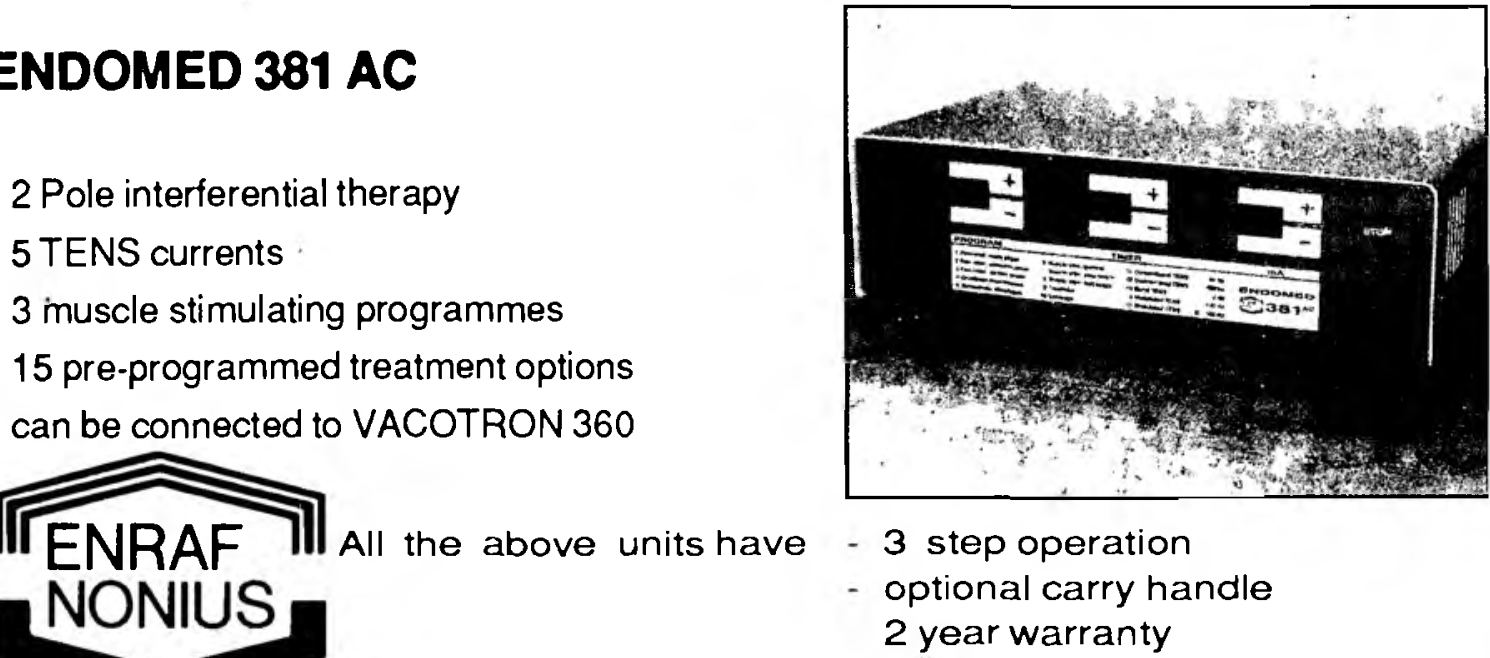

3 step operation

optional carry handle

2 year warranty

TUV/GS and IEC 601-1 approval

Head Office: Delft House, 376 Rivonia Boulevard, Sandton. Tel (011) 803-9320/1/2/3 Telefax (011) 803-7085 Cape OHtice: 18 Vlei Str. Bellville, Cape. Tel (021) 946-4560/1 Telefax (021) 948-8401 
measurements were also easy to perform, required no special setting other than an illuminated walkway and were obtained with minimal time commitment on the part of the researcher. It is also expected that since the measurements are easily standardised that different observers would achieve a similar result.

However, in spite of significant measurement reliability and statistically significant improvements on a validated subjective index in three cases undergoing exercise therapy, the trend towards improvement in walking time in this subgroup of patients $(t=2.05, n-3, p-0.17)$ fell within the limits of normal variability of the measurement, suggesting poor measurement sensitivity. Although this has been implied for rheumatoid arthritic trials ${ }^{16}$, a small sample with limited power might reduce the ability to detect statistically significant differences in walking time before and after treatment, even if these actually exist, resulting in a beta error. In addition, the duration or intensity of the current strengthening program might have been insufficient.

Improvements in walking speed might also be expected to be more evident following the effects of treatments such as unicompartmental prosthetic knee replacements as suggested by the findings of Mattsson et al for 19 of 20 patients with moderate knee OA. They not only found an individual change in the parameters of self-selected walking speed for these patients postsurgery, confirming the sensitivity of the measurements, but also reported that the improvements in walking time were correlated with favourable changes in single limb support time, indicating measurement validity. This was in line with the findings of Stauffer et al ${ }^{13}$ who reported significant correlations between the variable of gait velocity and those of pain, range of motion, walking distance, sitting and rising from a chair and instability in 65 patients with degenerative joint disease examined preoperatively. Based on a study of patients with OA of the knee who underwent total knee arthroplasty, Collopy et al ${ }^{25}$ postulated that strength of the knee extensor muscles was related to free walking velocity which they reported was a sensitive indicator of functional performance.

However, the usefulness of walking time measurements for measuring clinical improvements in knee function was not supported for persons with knee OA following drug therapy despite clinical improvements in rest pain, knee range of motion and stiffness. Lack of measurement sensitivity might also serve to explain the outcome of a controlled study of fenoprofen therapy in geriatric patients with knee $\mathrm{OA}$ in which the time required to walk 50 feet was the only parameter demonstrat- ing insignificant improvement ${ }^{1}$. This was also the finding with post-test baseline comparisons in free velocity reported by Peterson et al $^{19}$ which failed to achieve significance when intervention $(n=47)$ and control groups $(n=44)$ of patients with knee OA were compared after an eight-week program of physical exercise, which included strengthening. Thus, while walking tests appear useful for assessing the locomotor handicap occurring in OA of the knee postsurgery ${ }^{3,6,7}$ doubt arises as to their utility for assessing the efficacy of more conservative therapies such as drugs and exercise for the treatment of this condition. This was also the conclusion reached by Grace $e t a l^{16}$ for persons with rheumatoid arthritis.

These data suggest that inasmuch as the goal of physiotherapy for knee $\mathrm{OA}$ is to improve function, that due to their poor responsiveness, measurements of selfpaced walking may prove inaccurate for monitoring longitudinal changes in functional ability in this group of patients, despite their reliability, practicality and costeffectiveness. Pending further research using a larger sample, it is therefore recommended that to ensure important therapeutic changes are not overlooked in trials designed to evaluate efficacy for individuals with knee $O A$, validated tools such as the ISK be used concurrently for measuring functional capacity

\section{ACKNOWLEDGEMENTS}

This study was funded by The Arthritis Society, Toronto.

\section{REFERENCES}

1. McMahon F, Jain A, Onel A. Controlled evaluation of fenoprofen in geriatric patients with osteoarthritis. J Rheumatol 1976;2(Suppl):7682.

2. Cimmino M, Cutolo M, Samanta et al. Shortterm treatment of osteoarthritis: A comparison of sodium meclofenamate and ibuprofen. J Int Med Res 1982;10:46-52.

3. Schank JA, Herdman SJ, Bloyer RG. Physical Therapy in the multidisciplinary assessment and management of osteoarthritis. Clinical Therapeutics 1986;9(Suppl B):14-23.

4. Steiner ME, Simon SR, Pisciotta JC. Early changes in gait and maximum knee torque following knee arthroplasty. Clin Orthop 1988;238:174-182.

5. Blin O, Pailhous J, Lafforque P et al. Quantitative analysis of walking in patients with knee osteoarthritis: a method of assessing the effectiveness of non-steroidal anti-inflammatory treatment. Ann Rheum Dis 1990;49:990993.

6. Mattsson E, Olsson E, Brostrom L. Assessment of walking before and after unicompartmental knee arthroplasty. A comparison of different methods. Scand I Rehabil Med 1990;22:4550.

7. Mattsson E, Brostrom L. The physical and continued on page 8 ... effects in this population, such as radiographic and laboratory measures. The 
psychosocial effect of moderate osteoarthrosis of the knee. Scand / Relubil Mcd 1991;23:215-218.

8. Bellamy NW, Buchanan WW, Goldsmith CH ol al. Validation study of WOMAC: A health status instrument for measuring clinically important relevant outcomes to antirheumatic drug therapy in patients with osteoarthritis of the hip or knee. I Rhermatol 1988;15:1833-1840

9. Cowell HR. Editorial. Radiographic measurements and clinical decisions. I Bone Joint Surr 199();72A:319.

10. Falconer J, Hayes KW. A simple method to measure gait for use in arthritis clinical research. Arlirilis Carr Re's 1991;4:52-57.

11. Altman RD. Classification of discases: osteoarthritis. Somin Arllerilis Rh'um 1991;20(Suppl 2):40)-47.

12. Lequesne M, Mery C, Samson M it al. Indexes of severity for osteoarthritis of the hip and knee. Validation. Value in comparison with other assessment tests. Scand ) Rhouma10/ 1987;(Suppl 65):85-89.

13. Stauffer RN, Chao EYS, Gyory AN Biomechanical gait analysis of the disease joint. Clin Orlhop) 1977;126:246-255.

14. Shrout PE, Flciss JL. Intraclass correlations: uses in assussing rater reliability. Psycho/ /iull $1979 ; 86: 42()-428$.

15. Hayes KW. The effect of the awareness of measurement error on physical therapists' confidence in their decisions. Phys Ther 1992;72:515-531.

16. Grace EM, Gerecz EM, Kassam YM ot al 50-foot walking time: a critical assessment of an outcome measure in clinical therapeutic trials of antirheumatic drugss. $\mathrm{Br} / \mathrm{R} / \mathrm{r}^{2} \mathrm{~m}$ mul ol 1988;27:372-374.

17. Pincus T, Brooks Rh, Callahan LF. Reliability of grip strength, walking time and button test performed according to a standard protocol. / Rh'umatol 1991;18:997-100()

18. Jvarsson I, Larsson LE. Gait analysis in patients with gonarthrosis treated by high tibial osteotomy. Clin Orl/top 1989;238:185-190.

19. Puterson MCE, Kovar-Toledano PA, Otis JC d al. Elfect of a walking program on gait characteristics in patients with osteoarthritis Arlinitis Carr Re's 1993;6:11-16.

20. Lankhorst GJ, van der Standt RJ, van der Korst JK. The relationships of functional capacity, pain, and isometric and isokinetic torque in osteoarthrosis of the knee. Scomb ) Relub Mell 1985;17:167-172.

21. Collopy MC, Murray MP, Gardner GM al al Kinesiologic measurements of functional performance before and after gerometric total knee replacement. Onc-year follow-up of twenty cases. Clin Orflop' 1977;126:196-212.

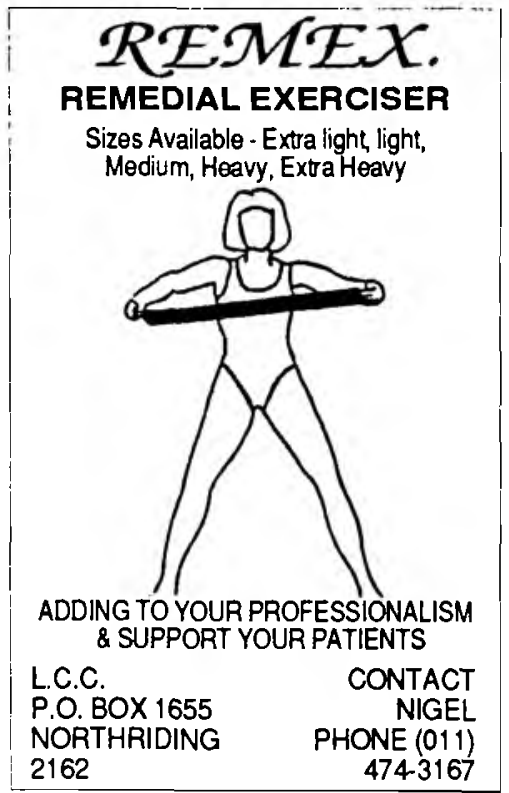

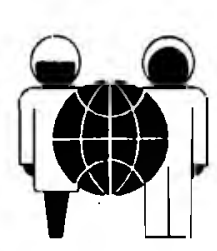

O'GRADY PEYTON international
OFFICES:
BOSTON
470 Atlantic Ave
8th Floor
Boston, MA 02210
Tel: (617) 482-5655
Fax: (617) 482-1551

\section{SAVANNAH \\ 7370 Hodgson}
Mem. Drive
Savannah, GA 31405
Tel: (912) 353-9366
Fax: (912) 353-9341

\section{SOUTH AFRICA}
PO Box 766
Somerset West 7129
Cape Province
Tel: (024) 51-6114
Fax: (024) 852-5508

\section{USA CALLING \\ EXCELLENT OPPORTUNITIES $\$ 2,000$ SIGN-ON BONUS}

PHYSIOTHERAPISTS! O'GRADY-PEYTON INTERNATIONAL recruits close to 400 healthcare professionals each year for hospitals all over the USA. That's trust. That's experience! We know where the best jobs are! We know how to provide the best advice and support along each step of the way. We take care of all the licensing and visa processing (both $\mathrm{H}-1$ and Green Card).

We provide an excellent salary and benefits package which includes free furnished accommodation for a whole year, free flights at the beginning and end of your contract and free medical insurance.

We also offer a US\$2,000 (US Dollars) sign-on bonus to help you get started, purchase a car etc. You won't beat that.

Please call Eileen Bryans, telephone number (024) 51-6114 and we will take it from there. Personal interviews will take place as follows:

\section{Johannesburg: \\ Sandton Sun Hotel \\ Durban: \\ Elangeni Sun Hotel \\ Cape Town: \\ Ambassador Hotel Sea Point}

Monday, March 7

Tuesday, March 8

Wednesday, March 9

Thursday, March 10
Friday, March 11

Occupational Therapists - we would also like to hear from you! 